\title{
APPLICATION OF TOOL SCIENCE TECHNIQUES TO IMPROVE TOOL EFFICIENCY FOR A DRY ETCH CLUSTER TOOL
}

\author{
Robert Havey \\ Lixin Wang \\ DongJin Kim \\ Micron Technology Inc. \\ 9600 Godwin Drive \\ Manassas, VA 20136, USA
}

\begin{abstract}
Semiconductor manufacturing is a capital-intensive industry. How to utilize billions of dollars of equipment as efficiently as possible is a critical factor for a semiconductor manufacturer to succeed in stiff competition. Unlike operations management techniques, like planning and scheduling, which are proven to improve tool performance by controlling WIP (work-in-process) movement, tool science techniques focus on tool architecture, components and operations inside the tool. In this paper, we first studied process time behavior of a cluster tool and fixed inefficient process sequence. A Petri Net model was then created to determine the internal bottleneck component of the tool. Results indicated that tool science techniques helped improve tool efficiency and resulted in significant cost savings.
\end{abstract}

\section{INTRODUCTION}

Dry Etch is an expensive process used for obtaining deep vertical sidewall trenches or holes through various film structures. The Dry Etch process accounts for $\sim 8 \%$ of the total moves of a Fab. The toolset chosen in this project was picked because the tool was showing tri-modal process times for the same recipe and root cause could not be determined. The objective of this project was to determine root cause of the tri-modal process times, implement a solution and validate the solution correcting the tri-modal process time distribution. Additionally, we want to study tool movement behavior and determine any potential opportunities to improve tool processing times.

\section{DRY ETCH TOOL DESCRIPTION}

The Dry Etch tool that was analyzed consisted of four single wafer processing chambers, one high vacuum robot with two end effectors, two air locks, one ATM (atmospheric) robot with one end effector, one orientor and three loadports as illustrated in Figure 1. During steady state processing, a wafer will move from the loadport to the orientor via the ATM robot, than from the orientor to the air lock via the same robot. If there is a processed wafer in the air lock, the ATM robot will remove the wafer and place it back in the loadport.

After the wafer is inserted into the air lock, the air lock pumps down to vacuum and waits for a chamber to become available. When a chamber becomes available, the high vacuum robot will insert the processed wafer into the air lock first, and then remove the unprocessed wafer from the air lock. At this time, the air lock will vent back to atmosphere. The unprocessed wafer will be inserted into the chamber for processing. 


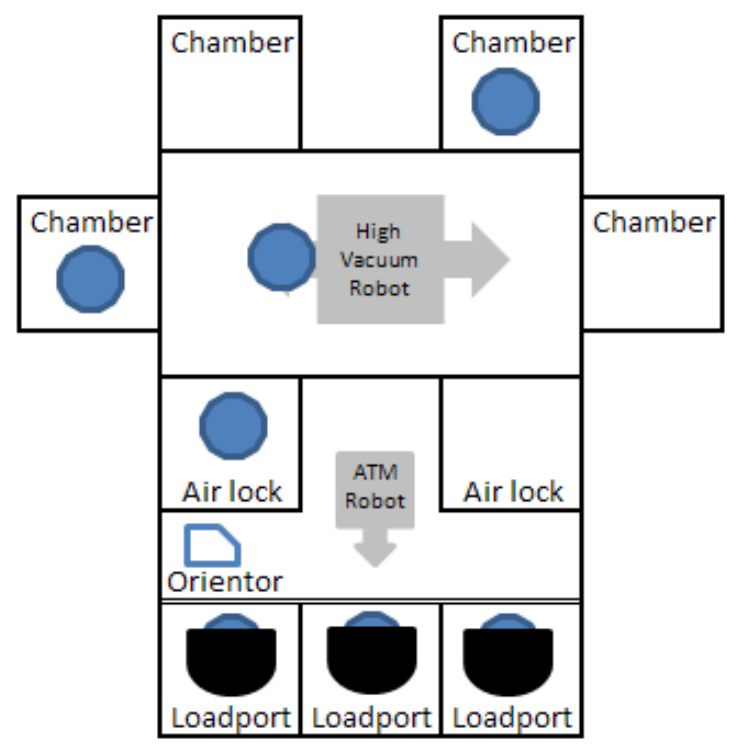

Figure 1: Representation of the Dry Etch tool

When the wafer completes processing in the chamber, the high vacuum robot will remove the wafer from the chamber and place it into the air lock that is pumped down to vacuum. The air lock will vent to atmosphere and then the wafer will be removed from the air lock via the ATM robot and placed back into the loadport it originally came from.

\section{LITERATURE REVIEW}

In the demanding environment of the cluster tool industry, it is very important for equipment manufacturers to design high performance new generation tools, continually improve the performance of existing designs, and increase the value of the equipment for their customers. Since cluster tools are complex mechatronic systems, there is a need among designers for understanding the various factors that influence throughput and their interactions (Burggraaf 1995). Some of these factors include the number of process chambers, the process times, the number and type of robots, the robot speeds, wafer scheduling methods, and many other variables. Tool science, in general, is to utilize industrial engineering techniques to tackle the above factors and improve tool operation efficiency with help from equipment and process engineers.

One important performance indicator for a cluster tool is process time, which is defined as the time a wafer is tracked into the tool until the time it is tracked out of the tool. Generally, for the same tool, with same wafer scheduling methods, process time distribution should be similar, if not the same. However, due to different input configuration by different engineers, it can be very different. According to our best knowledge, there have not been any publications related to this category.

Wafer scheduling methods, which determine sequence of wafer movements across chambers, robots and other tool components, play an important role in determining process time. There have been a lot of studies on wafer scheduling methods for cluster tools (Lee 2008). In this paper, we assume scheduling methods are fixed.

Some factors, e.g., number of process chambers, number and types of robots, robot speeds, are handled at design stage. However, it does not mean they are optimal in terms of process time for all fabs and all product mixes. To find the optimal combination of those factors require clear understanding of the interactions and effects of those factors on process time. Operational behaviors of cluster tools can be well modeled by Petri nets. A Petri net is a graphical and mathematical modeling framework for discrete 
event systems (Murata 1989). In a very early study of Petri Net for cluster tool, Petri net model is incorporated in a modified reachability analysis procedure that provides design and operation guidelines, in addition to evaluating throughput (Srinivasan 1998). Different Petri Net models and modeling methodologies for similar purpose can also be found (Zuberek 2001a, 2001b, 2004).

\section{TRI-MODAL PROCESS TIME DISTRIBUTION}

Several attempts were made to understand the root cause of the tri-modal process time distribution as seen in Figure 2. The Process Owner, Tool Owner and Dry Etch Planner attempted to determine the root cause, but all ideas that were explored did not work. The Industrial Engineering team was pulled in to determine if Tool Science Techniques would be able to analyze and determine the root cause.

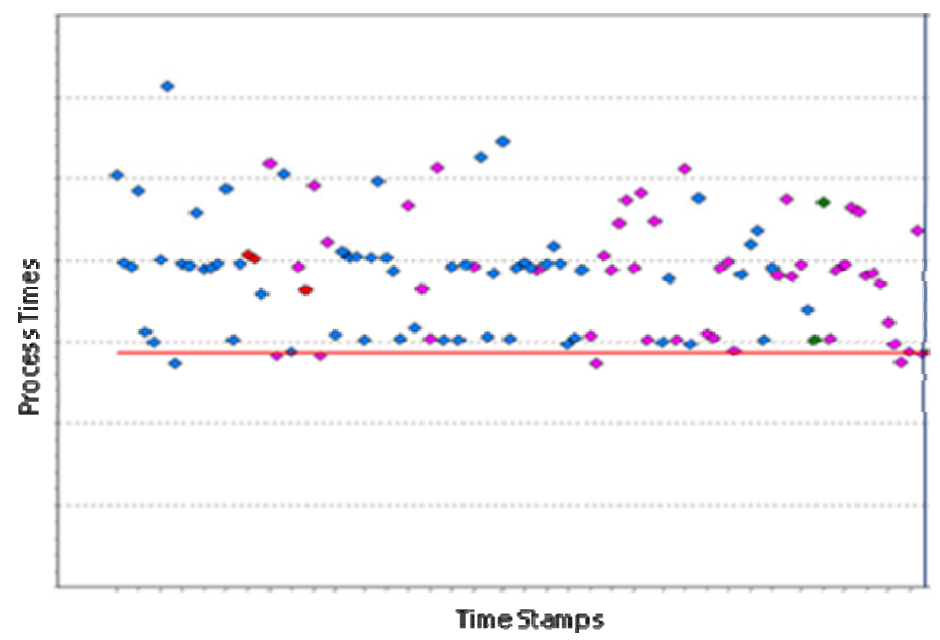

Figure 2: Tri-Modal process time distribution

\section{TOOL SCIENCE TECHNIQUES}

The Tool Science Techniques are a series of steps used to analyze a tool and understand how the tool works. The wafer movement through the entire tool is analyzed, the processing component of the tool is studied to determine what happens before, during and after wafer processing, and sequencing of the wafers through the tool is examined to determine the optimal wafer flow path through the tool.

The Dry Etch tool that was analyzed has:

- One Atmospheric Robot (ATM Arm)

- Two air locks (AirLock 1 In and AirLock 1 Out, and AirLock 2 In and AirLock 2 Out)

- One High Vacuum Robot with two end effectors ( EndEffector 1 and EndEffector 2)

- Four Process Chambers (PM1, PM2, PM3 and PM4)

\section{TOOL ANALYSIS}

To analyze the tool, the tool logs are pulled and critical component information is placed into a database so the data can be analyzed and organized. Once the data has been compiled and organized, the data can be formatted into Gantt Charts for analysis.

The Process Chambers (PM1 - PM4) were analyzed first to determine if the chambers were performing in an optimal manner. In Figure 3, the white space for PM1 - PM4 on the Wafer Location Gantt is the time that a chamber was not processing a wafer. The colored space shows the time the chamber was processing a wafer. 


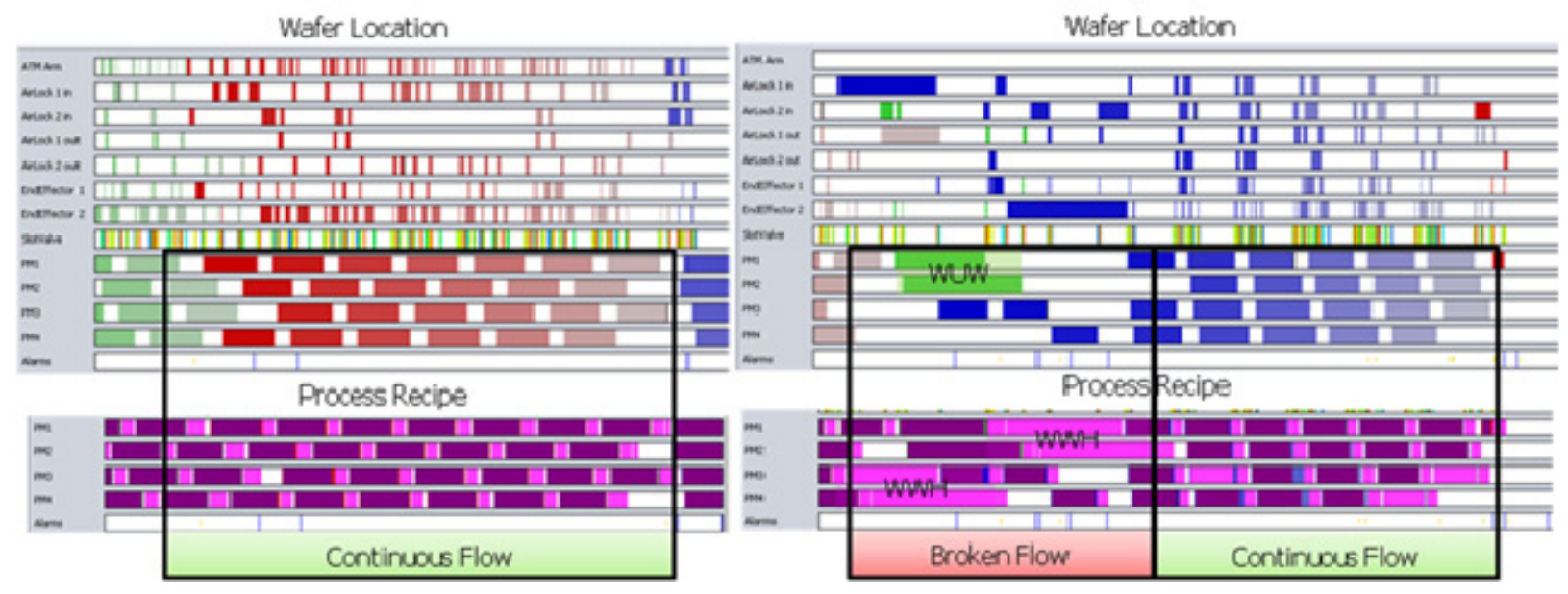

Figure 3: Gantt Charts - Normal processing tool vs. Offending tool

Looking at the Process Recipe Gantt, the colors show the time that the chamber was performing a function within the chamber. The maroon color is the actual processing of the wafer, which corresponds with the colors spaced in the Wafer Location Gantt. The pink color shows time that the chamber was prepping the chamber (waferless clean and leak check) before the next wafer can be processed.

In Figure 3, the left Gantt Charts show normal processing of wafers through the chambers (PM1 PM4). The colored bars are the same size and the white space is minimal. The right Gantt Charts shows has too much white space and the colored bars on the Process Recipe Gantt are not a consistent size. By analyzing the data that created this Gantt, the team was able to identify a potential root cause for the trimodal process time distribution.

On the right Process Recipe Gantt, there were two sections of abnormally long pink bars (chamber prepping). The logs showed that each chamber performed a specific chamber setup. It was also observed that when the setup was run, a wafer would get trapped on the End Effector (long blue bar on EndEffector 2). Since Process Time is calculated from Track Out of previous lot to Track Out of current lot, a wafer being trapped within the tool would cause the Process Time for the lot to be longer than the Modeled Process Time.

After identifying the setup issue, the Process Owner dug into the issue and concluded that the recipe on the tool was incorrect and the error could only be seen when the Process Owner stepped through the recipe on the tool. Once the error was corrected, the tool was allowed to run for a couple of days before additional data was collected. In Figure 4, the change to the recipe fixed the issue of the tri-model process times and the tool went back to running at the model process time, as indicated by the red line.

It can be seen that process time dropped significantly which resulted in improved tool efficiency. This can be converted to significant amount of savings on capital expense.

\section{PETRINET MODEL}

After the Gantt Charts were fully analyzed, a Petri Net Model was developed to determine what the current internal bottleneck of the tool is. To develop a Petri Net Model, the wafer movement through the tool must be fully analyzed and the amount of time the wafer spends within each section must be calculated. In Figure 5, the Petri Net Model was created for the Dry Etch tool that was analyzed. Based on the model, the internal bottleneck of the tool is the processing chambers (PM Loop), which was expected since this is the component of the tool that does the work on the wafer.

Based on this model, improvements made on any of the components within the PM Loop would improve the throughput of this tool. Improvements made on other components of this tool would not gain any additional throughput. 


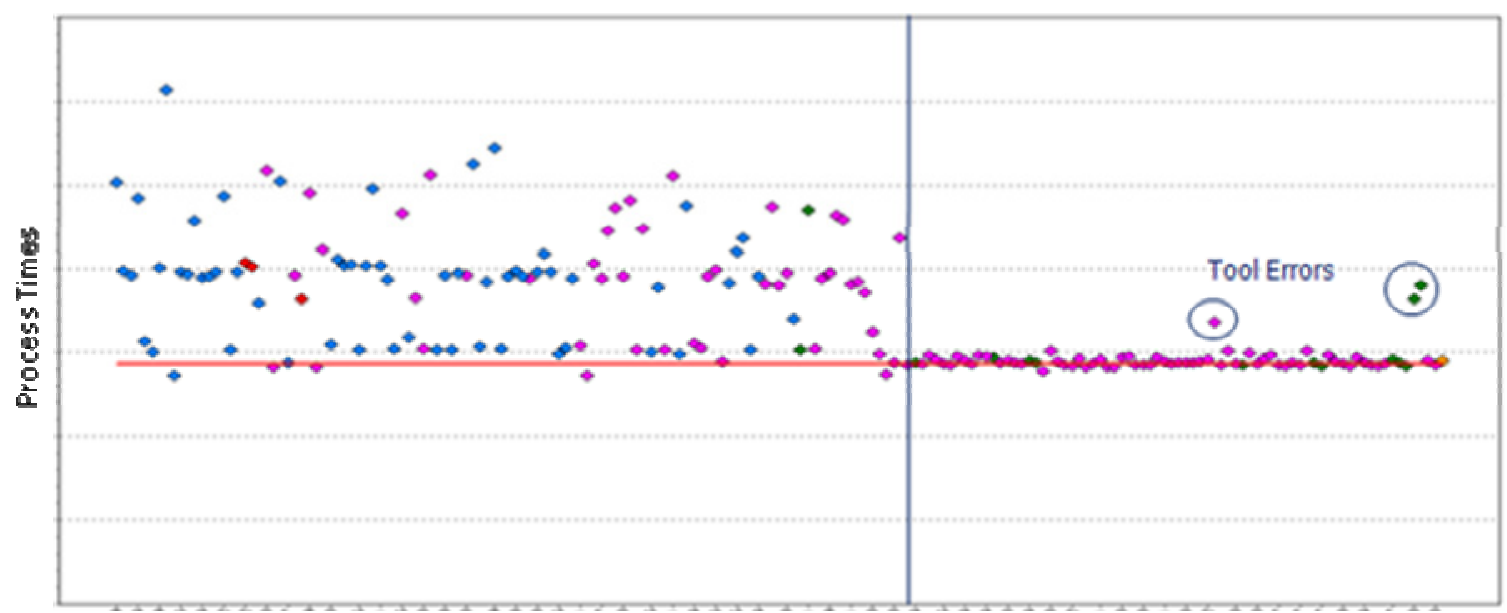

Time Stanps

Figure 4: Pre - Post Tri-Modal Process Time distribution

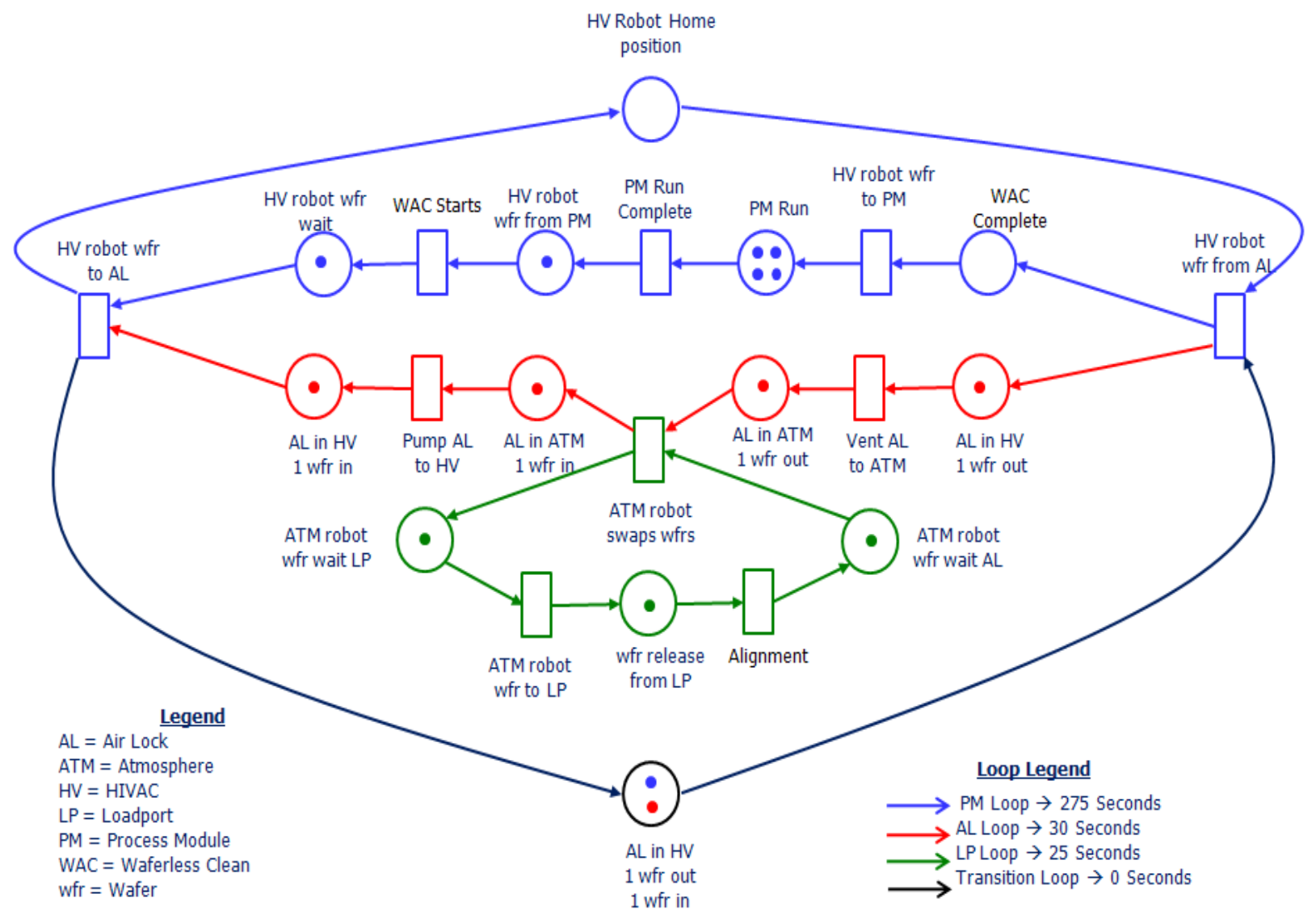

Figure 5: PetriNet Model for Dry Etch tool 


\section{CONCLUDING REMARKS}

In this paper, we utilized tool science techniques to study a Dry Etch cluster tool. Through observation on tool $\operatorname{logs}$ and other critical information, we were able to identify the root cause for the tri-model process time distribution. We also built a Petri Net model for the cluster tool and determined the movement bottleneck. Potential improvement opportunities are also identified through the Petri Net model.

The work described in this paper has contributed to significant capital savings for the fab. This proved that tool science techniques is a promising approach though the process involves manpower from crossteams and requires substantial data analysis.

\section{REFERENCES}

Burggraaf, P. "Coping with the High Cost of Wafer Fabs." Semicond. Int. 18(3):411.

Lee, T. E. 2008. "A Review of Scheduling Theory and Methods for Semiconductor Manufacturing Cluster Tools." In Proceedings of the 2008 Winter Simulation Conference, edited by S. J. Mason, R. R. Hill, L. Mönch, O. Rose, T. Jefferson, J. W. Fowler, 2127-2135. Piscataway, New Jersey: Institute of Electrical and Electronics Engineers, Inc.

Murata, T. 1989. "Petri Nets: Properties, Analysis and Applications." Proceedings of the IEEE 77(4): 541-580.

Srinivasan, R. S. 1998. "Modeling and Performance Analysis of Cluster Tools Using Petri Nets." IEEE Transactions on Semiconductor Manufacturing 11:394-403.

Zuberek, W. M. 2001a. "Timed Petri Net Models of Multi-Robot Cluster Tools." In Proceedings of 2001 IEEE International Conference on Systems, Man, and Cybernetics, 2729-2734.

Zuberek, W. M. 2001b. "Timed Petri Nets in Modeling and Analysis of Cluster Tools." IEEE Transactions on Robotics and Automation 17(5):562-575.

Zuberek, W. M. 2004. "Cluster Tools with Chamber Revisiting: Modeling and Analysis Using Timed Petri Nets." IEEE Transactions on Semiconductor Manufacturing 17(3):333-344.

\section{AUTHOR BIOGRAPHIES}

ROBERT HAVEY is an industrial engineer in the Industrial Engineering department at Micron Technology Inc., Virginia. He received B.S. in Industrial Engineering from Texas A\&M University and has done 11 years of semiconductor and Automated Material Handling System planning. His e-mail is rhavey@micron.com.

LIXIN WANG is an industrial engineer in the Industrial Engineering department at Micron Technology Inc., Virginia. He received B.S. in Mechanical Engineering from Tsinghua University, Beijing, China and Ph.D. in Industrial and Systems Engineering from Virginia Tech. His interest is modeling and simulation of semiconductor manufacturing systems. He is a member of INFORMS and IIE. His e-mail is stanleywang@micron.com.

DONGJIN KIM is an operations improvement manager in the Industrial Engineering department at Micron Technology Inc., Virginia. He received M.S. in Engineering Management Queensland University of Technology, Australia and has performed 14 years of simulation and modeling experiences in semiconductor industry. He is a member of INFORMS. His e-mail is djkim@micron.com. 